strips of flannelette in the following solutions respectively: pot. permanganate, 1:5000 ; hydrargyrum perchloridum, $1: 1000$; tinctura benzoin. co. ; aqueous solution of borsal (equal parts of salicylic acid and boracic acid); and boracic acid saturated solution. These strips were dried, laid in a row, and $\mathbf{X}$ rayed. The strip soaked in tinct. benzoin. co. showed the densest shadow, while that treated with the borsal was next best in density. As a medium similar to borsal seemed to be the easiest one to work with I decided to experiment with a modification of this. Salicylic acid by itself is not very soluble in water. If, however, borax be added it is readily soluble. The solution I used was : $\mathbf{R}$ Boracie acid, gr. $x v$. ; salicylic acid, gr. $x \nabla$. ; borax, gr. xxiv.; aq. ad $z i$. This solution is referred to hereafter as "B.S.B."

1. To ascertain if B.S.B. is impermeable to $X$ rays.-I had a case of acute extravasation of urine with urethral stricture, the scrotum being the size of a child's head. Ten days after the perineal cystostomy I soaked a piece of sterile white gauze in B.S.B. solution and introduced it into the deep sinus. The scrotum (with the gauze packed in) was X rayed, when the shadow was easily distinguished.

2. To ascertain if B.S.B. has antiseptic properties.-The B.S.B. was made up as if it were an ordinary medicine-i.e., the dispenser made up the mixture with the ship's tap-water in an ordinary medicine bottle. Nothing was sterilised. Three tubes of agar were obtained, together with some pus from a septic wound which contained staphylococcus and streptococcus. The agar tubes were sown as follows: Tube $a$ with 1 platinum loopful of pus only; Tube $b$ with 1 platinum loopful of pus and 1 platinum loopful of the B.S.B. solution and Tube $c$ with 1 platinum loopful of the B.S.B. solution only. At the end of 48 hours' incubation the results to the naked eye were: Tube $a$, profuse growth; Tube $b$, growth, but nothing like so much as in.Tube $a$; Tube $c$, no growth whatever. Therefore, subject to confirmation, it may be assumed that B.S.B. solution has some antiseptic properties.

3. To ascertain if B.S.B. is non-irritating to the tissues. -The piece of gauze soaked in B.S.B. solution, packed into the scrotal sinus, as mentioned previously, was allowed to remain for 24 hours, when it was removed. It caused no discomfort, there was no caustic effect, and I think it materially helped granulation.

4. To ascertain if B.S.B. is non-irritating to the wearer's skin.-I soaked a woollen undervest in the solution, and after it was dry I wore it continuously for five days. During this period I was perspiring profusely in a superheated theatre while operating. It produced no discomfort whatever, nor did any rash appear. The perspiration was absorbed from the undervest normally after I had ceased perspiring.

My reason for publishing such an incomplete note is the difficulty of doing experimental work on board ship, and it will be some considerable time before I can do anything further. To only see the sepsis following some of these dreadful shell injuries is quite sufficient to stimulate one to try to think of prophylactic measures.

The experiments detailed require confirmation; most probably other substances which will answer the required purpose better will be suggested. I am hoping that Surgeon-General Sir William Watson Cheyne will think the subject worth following up.

Note by Surgeon-General Sir W. Watson CheYne.

I received a copy of the essential part of this paper from Staff-Surgeon Willan early last August, but was not at that time able to take up the question as he wished me to do. The suggestions are certainly most important ones, and even if only one of them could be realised in practice it would be a great help to surgical treatment. There is no doubt that the retention of pieces of dirty clothing in wounds is one of the most potent causes of serere sepsis, and the possibility of removing them early would be a great advance; this would be still more valuable if the opaque substance were itself antiseptic, or at least carried an antiseptic substance along with it in to the cloth.

Since I heard from Staff-Surgeon Willan I have made many inquiries and have thought much about the subject, with the result that $I$ came to the conclusion that a research by any one individual was not likely to be fruitful, as so many processes and sciences were involved; at any rate, $I$ did not feel capable of carrying out such a research myself. Hence I placed Staff-Surgeon Willan's suggestions before the Board of Scientific Studies; and I learn that it was much interested, and will, I hope, reach some satisfactory conclusion.

\section{THE TOXIC ACTION OF DILUTE PURE SODIUM CHLORIDE SOLUTIONS ON THE MENINGOCOCCUS.}

\author{
BY CRESSWELL SHEARER, M.D., D.So, F.R.S. \\ MILITARY HOSPITAL, DEVONPORT. \\ (Report to the Medioal Research Committee.)
}

Is experimenting during the past season with a large number of freshly isolated strains of the meningococcus it was noticed that almost all of them were killed when placed for a short time in dilute pure sodium chloride solutions. The action of sodium chloride is most toxic to the meningococcus when the concentration of the salt is not below 0.85 per cent. and not much above 0.9 per cent. $\mathrm{NaCl}$.

It was found that freshly isolated meningococci were more vulnerable than old laboratory cultures. While old laboratory cultures could sometimes resist the action of pure 0.85 per cent. NaOl for three or four hours, freshly isolated strains seldom resisted its action for more than 20 minutes.

It seemed remarkable that $\mathrm{NaCl}$ should be toxic for the meningococcus in that concentration to which this germ is accustomed in the fluids of the body. It is strange, moreover, it should be so sensitive to the action of sodinm chloride, while at the same time it is able to resist for many hours the action of distilled water.

To demonstrate the toxic action of saline solution on the meningococcus, it is highly important to observe certain conditions in performing the experiment. It is essential, in the first place, that the $\mathrm{NaCl}$ used should be free from any impurity. In the second place, the meningococci must be added to such a quantity of the saline solution that any trace of salts brought over with them from the culture-medium will have no appreciable effect in antagonising the action of the NaCl. Thirdly, it is important that the saline is not unduly diluted below its proper toxic strength by the addition of too large a quantity of the emulsion containing the germs. In the fourth place, no agglutination of the germs into masses or clumps in the saline must be allowed. The saline is then unable to act on the germs in the interior of these clumps, so that all are killed. Care must be taken to shake up the germs thoroughly in the saline and avoid all clumping as much as possible.

I have found, as a general rule, from a large number of experiments that one or two drops of an emulsion (of 5000 million meningococci to the cubic centimetre in distilled water) is the proper quantity of emulsion to be added to 2 cubic centimetres of 0.85 per cent. saline to demonstrate the toxic action of pure $\mathrm{NaCl}$ effectively on the meningococcus.

The toxic action of a 0.85 per cent. $\mathrm{NaCl}$ solution on the meningococcus can be readily antagonised and rendered harmless by the addition of a trace of some bivalent

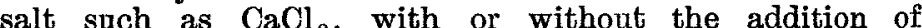
a small quantity of $\mathrm{KCl}$. This is clearly shown by the following experiment, which has been repeated many times.

A 24-hour culture of a recently isolated strain of meningococcus "Lake" was emulsified in distilled water, a fairly thick emulsion being prepared (about 5000 million cocci to the c.c.). Into four sterile test-tubes capable of being placed in a centrifuge the following solutions with $25 \mathrm{c.mm}$. of the meningococcus emulsion were placed as follows :-

(1.) 2 c.c. sterile $0.85 \% \mathrm{NaCl}$.

(2.) $2, \quad, \quad 0.85 \% \mathrm{NaCl}+0.004$ c.c. $\frac{\mathrm{M}}{1} \mathrm{CaCl}_{2}$.

(3.) $2, \quad, \quad 0.85 \% \mathrm{NaCl}+0.004$ c.c. $\frac{\mathbf{M}}{1} \mathrm{CaCl}_{2}+001$ c.c. $\frac{\mathbf{M}}{1} \mathrm{Kal}$.

\section{(4.) $2, "$ distilled water.}

Each tube was then thoroughly shaken to ensure thorough mixing of the solutions and the emulsion of cocci. They were put in the incubator at $37^{\circ} \mathrm{C}$. for an hour and a quarter. They were then taken out and centrifuged hard for 15 minutes, and the deposit in each tube planted out separately in sterile fashion on a chocolate plate. ${ }^{1}$ Fig. 1 shows the growth obtained on this plate after incubation for 24 hours at $37^{\circ} \mathrm{C}$

An examination of Fig. 1 shows that the meningococcus emulsion placed in the pure 0.85 per cent. $\mathrm{NaCl}$ - that is, the deposit from Tube (1) has failed to grow, and that this 
quarter of the plate (marked I.) planted out with this deposit is quite free of colonies. The cocci have been killed by the saline. In the opposite quadrant of the plate to this (II.), which has received a similar quantity of emulsion in 0.85 per cent. $\mathrm{NaCl}$ with the addition of a trace of $\mathrm{CaCl}_{2}$, a thick heavy growth of the meningococcus has taken place, covering the entire surface of this quarter of the plate. The $\mathrm{CaCl}_{2}$ here has completely antagonised the toxic action of the $\mathrm{NaUl}$. In Quadrant III., where the saline has received the same quantity of $\mathrm{CaCl}_{3}$ as II., but also a little $\mathrm{KCl}$, growth is still thicker (not very well shown in the photograph). In IV, where the germ was simply allowed to stand for an hour and a quarter in distilled water, growth is good, and the colonies cover closely the entire surface of this quarter of the plate.

This experiment clearly demonstrates the four following points :-

1. The toxic action of a pure 0.85 per cent. $\mathrm{NaCl}$ solution on the meningococcus.

2. The antiagoniatic action of a trace of $\mathrm{CaCl}_{2}$ solution over the toxic action of the $\mathrm{NaCl}$.

3. The accelerating action of $\mathrm{KCl}$, when added to $\mathrm{CaCl}_{2}$ in antagonising the toxic action of $\mathrm{NaCl}$.

4. The relatively harmless action of distilled water on the meningococous.

FIG. 1.

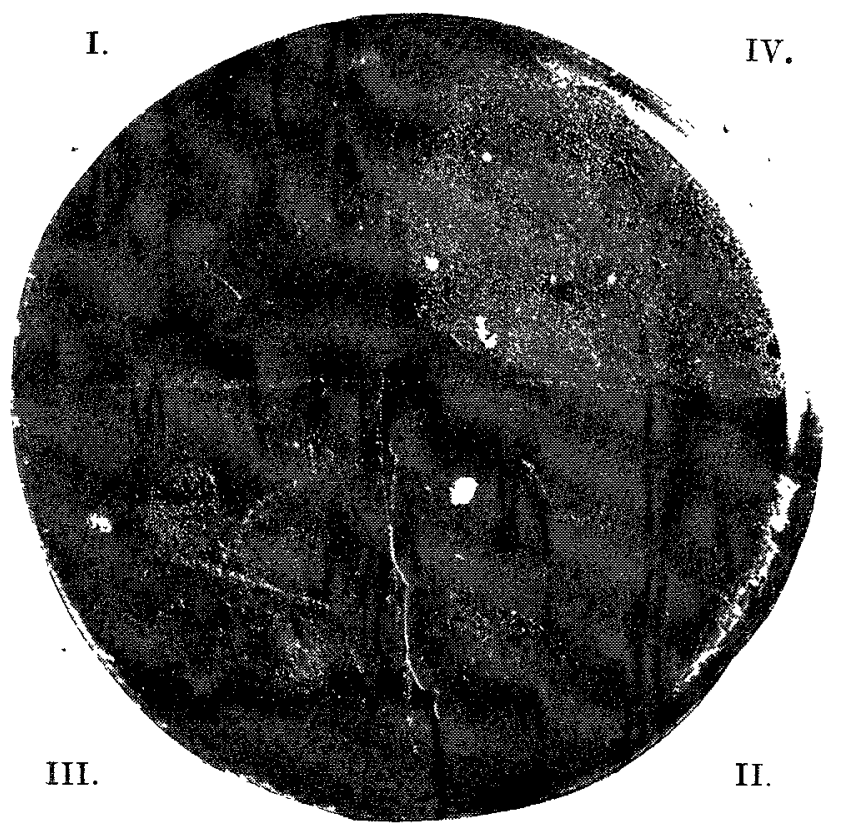

Photograph of the growth obrained on a plate of Crowe's chocolate medium after 24 bours incubation at $37^{\circ} \mathrm{C}$., showing the toxic action of a pure 0.85 per cent. NaCl solution on the meningococcus and the antagonistic action of a trace of $\mathrm{CaCl}_{2}$. I. Portion of plate planted out with emulsion of the meningococcus in 0.85 per cent. Nacl solution. No growth has taken place, all organisms being killed. II. Portion of plate planted ouf with a similn $r$ quantity of killed. II. P.85 of plate planted our with emulsion in 085 per cent. NaOl + a trace of $\mathrm{CaCl}_{2}$, here the $\mathrm{CaCl}_{2}$ has antagonised the toxic action growth cif the meningococcus. Ir. Portion of plate planted out with a similar quantity of emulsion in 0 s per cent $\mathrm{NaCl}+\mathrm{CaCl}_{2}+\mathrm{KCl}$ with a resulting thick heavy growth, more than in II. IV. Portion of plate planted out with a similar quantity of meningococcus emulsion which had been allowed to stand for an hour and a quarter in distilled water; good growth.

So definite is this toxic action of 085 per cent. $\mathrm{NaCl}$ solution on the meningococcus, that it was found possible to make use of it very successfully to destroy all meningococci outside or attached to the surface of leucocytes, by simply washing these several times and allowing them to stand for a few hours in a small bulk of pure saline. ${ }^{2}$ There is no doubt that, in this toxic action of dilute $\mathrm{NaCl}$ solution on the meningococcus we are dealing with the poisonous action

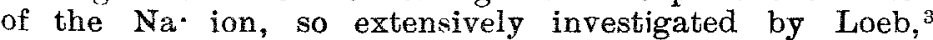
Wasteneys, ${ }^{4}$ Osterhout, ${ }^{5}$ and others.

It is interesting to find that in the case of the meningococcus. as these investigators have found for other forms of life, this toxic action of $\mathrm{NaCl}$ is confined to relatively dilute solutions. In the case of the meningococcus it is essential

"See paper by Shearer and Crowe: The Rôle of the Phagocrte in Cerebro-spinal Meningitis, Proc. Roy. Soc., London. (To appear shortly.)

3 Loeb: Collected Papers, Pt. 2, Univ. Chicago, 1906.

4 Loeb and Wasteneys: Jr. Bio. Cnem., rol. xxi.. 1915

Osterhout: Zeit. f. physhl. Chem., vol. lxx., 1910. that the concentration of the $\mathrm{NaCl}$ should not be increased much beyond 0.9 per cent., as after this point its toxic action rapidly decreases. The use of a 15 per cent. $\mathrm{NaCl}$ solution (one of the standard strengths of this salt employed in opsonic work) is without almost any tnxic action on the meningococcus, as shown by the following experiment.

FIG. 2.

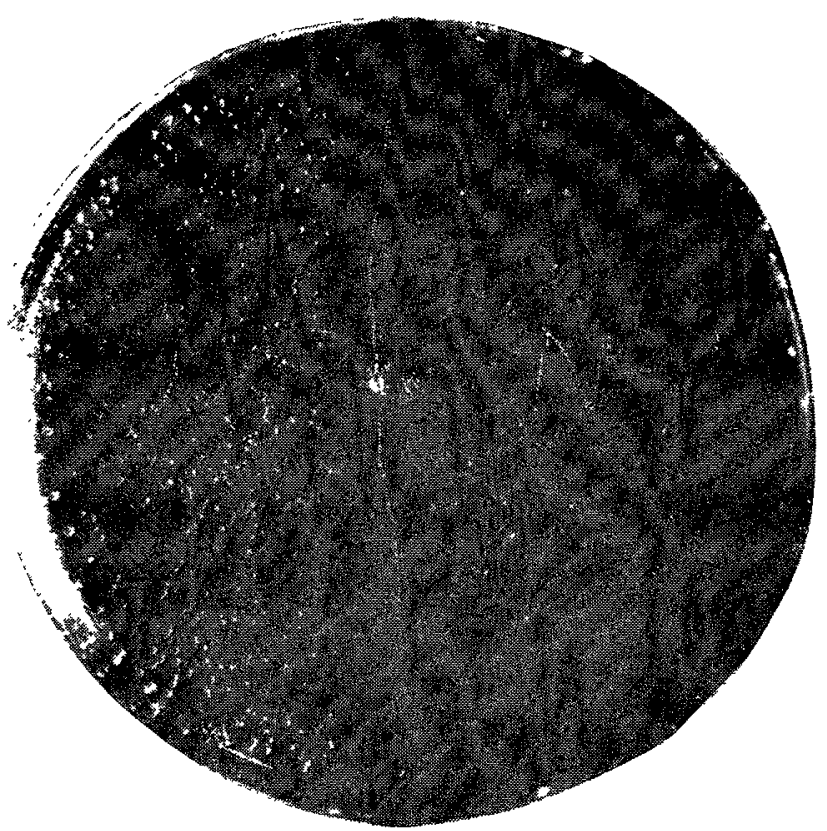

Showing the toxic action of 0.85 per cent. $\mathrm{NaCl}$ solustion on the meningococcus, in distinction to the relatively harmless action of a 1.5 per cent. solution of the same salt.

A fairly thick emulsion of a 24 hours' culture of meningococcus "Pryor" was made (about 5000 million cocci to the c.c.). To 4 c.c. of a pure 1.5 per cent $\mathrm{NaCl}$ solution $25 \mathrm{c.mm}$. of this culture in distilled water was added and thoroughly mixed. To $4 \mathrm{c}$ c of 085 per cent. $\mathrm{NaCl}$ solution a similar quantity of the same emulsion was added and mixed. The two solutions were placed in the incubator at $37^{\circ} \mathrm{C}$. for an hour. They were then taken out and centrifuged and the deposit planted out separately on the surface of a chocolate plate, as shown in Fig 2. This figure shows the resulting growth obtained on this plate after

FIG. 3 .

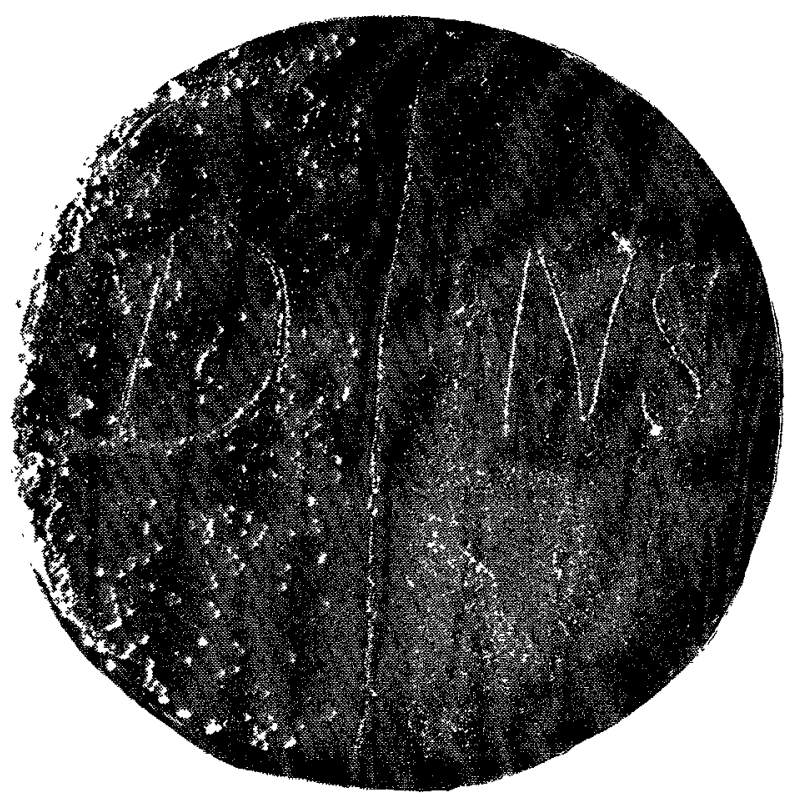

Showing the action of distilled wat $r$ in failing to kill the meningococeu after 24 hours. D, Distilled water portion. N.S., 0.85 per cent. NaC which has killed the germs.

24 hours incubation at $37^{\circ} \mathrm{C}$. The 0.85 per cent. NaCl solution (marked $\mathbf{N}$ in the plate) has killed the meningococcus, while a good growth has been obtained on that half of the plate inoculated with a similar quantity of the same emulsion which had been exposed to the action of a 1.5 per cent. solution. 
I should like to draw attention, finally, to an experiment made to determine the length of time the meningococcus may remain alive in pure distilled water, as compared with 0.85 per cent. $\mathrm{NaCl}$ solution. Into 4 c.c. of distilled water, and 4 c.c. of 0.85 per cent. $\mathrm{NaCl}$ respectively, $25 \mathrm{c} . \mathrm{mm}$. of a meningococcus emulsion was placed. The two solutions were then incubated for 24 hours at $37^{\circ} \mathrm{C}$., centrifuged down for an hour and planted out separately on a chocolate plate. In Fig. 3 is shown the resulting growth on this plate after 24 hours at $37^{\circ} \mathrm{C}$. The saline, as usual, has killed the germs; while a considerable number of those in the distilled water have survived and have given rise to a fair number of colonies. This experiment clearly demonstrates the power of the meningococcus to resist the action of distilled water.

\section{Antedical Socretics.}

\section{ROYAL SOCIETY OF MEDICINE.}

\section{CLINICAL SECTION.}

\section{Exhibition of Clinical Cases.}

A MEETING of this section was held on Nov. 10th, Sir Frederic Eve, Vice-President, being in the chair.

Dr. F. PARKes Weber showed a case of Thrombo-angiitis Obliterans (Non-syphilitic Arteritis Obliterans of Hebrews) affecting Three Limbs.

The man, aged 39, a Russian Jew, was the patient whose earlier history had been given in a paper on Thromboangiitis Obliterans. ${ }^{1}$ In 1908, when he was aged 31, his right leg was amputated below the knee for commencing gangrene. The disease had commenced about two years previously, with pains in the right leg on walking of the usual "intermittent claudication" kind. There was the ordinary history of cigarette-smoking (15 to 20 cigarettes a day). Macroscopically the arteries of the amputated limb appeared to be rather hypoplastic, as had been observed in some other cases of the disease. The posterior tibial artery showed organised thrombus, containing pigment granules (? hæmosiderin). The upper part of the posterior tibial artery showed no thrombosis, but much narrowing of the lumen from a kind of endarteritis obliterans. The lumen of the lower part of the posterior tibial artery was likewise not oblitera ted. About the vasa vasorum surrounding it there was considerable cell-infiltration. One of the venæ comites of the middle part of the anterior tibial artery was obliterated by organised thrombus containing pigment granules (? hæmosiderin). About that time (1908) the patient seemed to have true psoriasis of the tongue, and simple cutaneous psoriasis of his trunk, limbs, and scalp still persisted. There was no evidence of disease in the thoracic or abdominal viscera. The brachial systolic blood pressure (in both arms) was about $130 \mathrm{~mm}$. Hg. In July, 1912, owing to great and unyielding pain in the right stump, Dr. zum Busch amputated through the middle of the femur. In September, 1916, the patient was again admitted to hospital, having had for two years typical "intermittent claudication" on walking, in the calf-muscles of his remaining leg. For the last six months cr so he had suffered from pain and tendency to cyanosis, of the "pseudo-erythromelalgia" type, in the foot. There was no pulsation in the left dorsalis pedis artery. The left radial pulse could not be felt, and the artery was doubtless obliterated. The patient had for some time been subject to temporary feelings of coldness in that hand. The brachial systolic blood pressure in the right arm was $125 \mathrm{~mm}$. Hg. The Wassermann reaction was negative. The knee-jerk was present and the plantar reflex normal. He had not quite given up his old habit of cigarette-smoking. Since then, by rest in bed, \&c., there had been some improvement in regard to the turgid, erythromelalgia-like, congestion of the foot, but he needed morphine injections for sleep at night. He sometimes seemed to obtain relief by letting the leg hang down over the edge of the bed so that the foot became congested and purplish.

Dr. Weber also showed a case of Progressive Spinal Muscular Atrophy (Duchenne-Aran) following Electric Shock with Positive Wassermann Reaction.

The patient presented the appearance of great muscular atrophy of both upper extremities and of the muscles connected with the shoulder-girdle and thorax. There was great loss of power in the shoulders and arms. He

1 Quarterly Journal of Medicine, July, 1916 (ix., last paragraph on p. 296). could raise his left hand to his mouth, but could hardly raise his right hand. The wasting occurred first and was particularly noticeable in the intrinsic muscles of the hands. In both hands there was some contracture, more in the left than in the right. The atrophied muscles of the hands reacted very badly to electrical stimulation, the thenar muscles giving no definite reaction at all. His knee-jerks were exaggerated and, though he walked well, his gait was slightly spastic. There was no ankle clonus. The plantar reflexes were extensor. About March, 1910, he accidentally received a severe electric shock across the upper part of his body "from his left to his right hand" (750 volts, direct current). At first he seemed to suffer no ill-effects from it, but about 16 months afterwards he noticed that there was considerable wasting in his hands. The muscular atrophy slowly progressed until a few years ago.

There was no suspicion of any saturnism in the case and no history of syphilis could be obtained, but the Wassermann reaction had on two occasions been positive. The disease was clearly progressive spinal muscular atrophy. As usual, the patient was a male, of middle age when the first symptom was observed, and the hands were the first parts affected. 'l'hough he (Dr. Weber) had never heard of the disease following an electric shock, it was acknowledged to follow occasionally various kinds of traumata and shock, and he had been informed that in the present war it had been known to occur after "shell-shock." It was possible that a syphilitic taint occasionally acted as a predisposing cause by diminishing the resistance of the central nervous system.

Dr. WEBER also showed a case of Chorea Rhythmica in a man.

The patient, a man aged 38, had rhythmic movements of the neck and both arms which, under observation, became increased in rate, amplitude, and force. They quieted down somewhat when he was left to himself, and ceased altogether during sleep. Their rate was usually about 100 per minute. The movements became more violent when anyone tried to restrain them by force in any way or when the patient himself caught hold of a rail in endeavouring to walk. He said that he could not walk, but he could move his lower extremities when sitting or lying down. He gave one to understand that he could speak only in a very slow and broken (jerky) manner, as if battling against some obstruction in articulation. On the other hand, he could eat, drink, and dress himself fairly well when left alone without any assistance. He was not losing weight, and was apparently free from any visceral disease. His knee-jerks were exaggerated; no ankle clonus could be obtained plantar reflexes were flexor. The speech difficulty commenced in December, 1915, and about February, 1916, he gradually became unable to walk. The chorea rhythmica apparently began about July, 1916.

Obviously, psychical factors played an important part in the case.

Mr. P. B. Roth showed a case of Enlargement of the Lower Jaw (? Leontiasis Ossea).

The patient, a woman aged 56, was first seen in September of this year, complaining of pain in the lower jaw. She stated that in July she had several teeth out because of abscesses, and since then the gums seemed to have swollen. She complained at times of a shooting pain like a hot needle on the left side of her chin. Ten years ago, while carrying a heavy flower-pot in both arms, she stumbled, and fell with her chin against the pot. Her chin was badly bruised, and since then it had been getting larger. Examination showed a remarkable enlargement of the chin and lower jaw; the enlargement was more or less uniform, though the left side was rather more prominent than the right. All the lower teeth except a molar at the back had been removed. The alveolus was double or treble its normal size. A radiogram taken by Dr. W. Ironside Bruce showed a general enlargement of the horizontal ramus on each side.

Mr. RoTH also showed a case of Fracture of Both Patellæ in a woman. That on the right side was caused indirectly and had occurred recently; union had followed operation. The fracture of the left patella was due to direct injury. It took place eight years ago, the patient seeking no treatment but resting in bed for two months. It was a Y-shaped fracture, the patella being separated into an upper and outer and two lower and inner fragments. There was a gap of $2 \frac{1}{2}$ inches and no sign of bony union. Although the quadriceps had been functionless for eight years there was no contraction of the hamstrings.

Dr. H. BATTY SHAW showed a case of Congenital Heart Disease.

The patient, a man aged 32 , had from birth suffered from dyspnoa and cyanosis. A murmur corresponding to patency 